\title{
Soutien de l'organisation de la vie quotidienne chez les personnes souhaitant mourir à la maison
}

\section{Manon Guay}

Ergothérapeute, Ph. D., Professeure agrégée, École de réadaptation de la Faculté de médecine et des sciences de la santé de l'Université de Sherbrooke

Chercheure au centre de recherche sur le vieillissement, Sherbrooke, Canada

\section{Claudia Talbot-Coulombe}

Ergothérapeute, M. Sc., professionnelle de recherche au centre de recherche sur le vieillissement, Sherbrooke, Canada

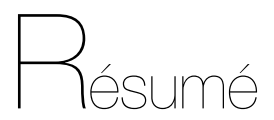

Soutien de l'organisation de la vie quotidienne chez les personnes souhaitant mourir à la maison - Bien que la majorité des personnes en soins palliatifs souhaitent mourir à leur domicile, peu d'entre eux voient leur souhait se réaliser. L'organisation du quotidien est l'un des obstacles au décès d'une personne à la maison. Cet article positionne l'ergothérapeute en tant qu'expert de l'aménagement de l'environnement domiciliaire aux (in)capacités des personnes, pour minimiser les situations de handicap et promouvoir la participation à des occupations porteuses de sens en fin de vie. L'histoire d'Hélène voulant prendre un bain pour soulager ses souffrances illustre que l'ergothérapeute soutient l'organisation de la vie quotidienne de la personne pour que sa vie et sa mort à la maison soient signifiantes et qu'elles se réalisent en cohérence avec ses aspirations.

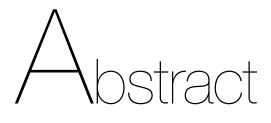

Supporting people who wish to die at home in the organization of their day-to-day living - Although the majority of people receiving palliative care wish to die at home, few see their wish realized. The organization of their day-to-day living is one of the obstacles to people dying at home. This article positions the occupational therapist as an expert in adapting the home environment to people's (in)abilities, in order to minimize situations of disability and encourage their participation in meaningful occupations at the end of their life. Hélène's story of wanting to take a bath to relieve her suffering is an example of how the occupational therapist supports people in the organization of their day-to-day living, so that their life and death at home are meaningful and are carried out in line with their wishes.

\section{Introduction}

Une grande proportion de la population nordaméricaine et européenne souhaite mourir à domicile [1,2]. Or, la majorité des personnes décèdent en milieu hospitalier. Moins du tiers des Américains qui sont susceptibles, ou ayant reçu des soins palliatifs, meurent chez eux (32\%) [3]. Si la proportion est du même ordre de grandeur aux Pays-Bas (35\%), elle est beaucoup plus basse au Canada: $12 \%$ dans la province du Québec [4] et $9 \%$ en Ontario [5]. La France (22\%) et la Belgique (25\%) se situent entre les deux, avec environ le quart des personnes mourant à domicile [3].

Les obstacles freinant la réalisation du souhait d'une personne de mourir à la maison sont nombreux. Parmi les embûches identifiées se trouvent notamment le manque de connaissances des professionnels de la santé sur les soins et les services de fin de vie, ainsi que l'accès inéquitable [6]. 
En plus d'un manque de financement pour les soins palliatifs à domicile, ceux-ci sont offerts principalement la semaine, dans les milieux urbains [6]. L'organisation des derniers jours à domicile est aussi un des enjeux documentés. Par exemple, le temps insuffisant pour livrer l'équipement nécessaire au décès, ainsi que le manque de soutien, et le fardeau qui en résulte sur les proches en limitent l'avènement $[6,7]$.

Les bonnes pratiques auprès des personnes désirant mourir à domicile précisent l'importance d'être entouré d'une équipe interdisciplinaire en soins palliatifs pour aider à contrer les défis rencontrés $[8,9]$, mais ces pratiques demeurent minimales. Au Québec, Gagnon et coll. ont étudié l'offre de soins et de services en soins palliatifs à domicile et ils ont identifié que $25 \%$ des personnes ayant décédé d'une cause reliée au cancer n'avaient pas eu la visite d'au moins un professionnel de la santé quarante-deux jours avant leur mort [4]. Pourtant, mourir à domicile exige, entre autres, d'anticiper et de planifier le déclin fonctionnel inhérent à l'évolution de la maladie, et de s'intéresser aux dimensions matérielles qui en découlent. Les bouleversements occupationnels associés à la fin de vie requièrent une réorganisation du quotidien et la mise en place d'assistance humaine et technique.

Lergothérapie peut favoriser le maintien à domicile et l'organisation du quotidien pour que la personne en fin de vie puisse mourir chez elle. Plusieurs groupes revendiquent la place de l'ergothérapeute dans les équipes interdisciplinaires pour soutenir l'identification et apporter des solutions aux enjeux matériels et occupationnels de la fin de la vie [10-13]. Selon l'Association canadienne d'ergothérapie, les ergothérapeutes devraient revendiquer le droit de leurs clients de mourir avec dignité, entourés de leurs proches, dans l'environnement de leur choix [10]. Le but de cet article est de positionner le rôle de l'ergothérapeute en soutien à l'organisation du quotidien dans les bonnes pratiques pour les personnes désirant mourir à domicile.

\section{Hélène voulait prendre un bain pour soulager ses souffrances}

Hélène est atteinte d'un cancer. En plus d'une grande fatigabilité, ses jambes sont faibles dû aux métastases qui compriment sa moelle épinière (paraparésie). Elle est locataire avec son conjoint d'un appartement de $3 \frac{1}{2}$ p pièces, au deuxième étage d'un duplex de banlieue. Puisqu'elle ne peut pas monter et descendre l'escalier, elle est confinée à domicile. Des services d'auxiliaires sont offerts trois fois par semaine, mais son conjoint est le principal aidant. Une demande en ergothérapie est faite par l'infirmière de l'équipe de soins palliatifs à domicile parce qu'Hélène aimerait prendre un bain.

Lors de l'évaluation, l'ergothérapeute constate qu'Hélène se douche sur un banc installé dans la baignoire avec l'aide de l'auxiliaire trois fois par semaine, puisque sasseoir et se relever du fond de la baignoire sont des risques éminents de blessures pour elle et pour ses aidants. Elle se sent propre, mais elle désire tremper dans l'eau: (1) «J'ai mal partout. Je rêve de prendre un bain avec de la mousse. Mon conjoint avait l'habitude de me mettre de la musique et des bougies, puis de me préparer un bon bain. L'eau chaude ferait tellement de bien à mon dos! »

Durant l'évaluation, l'ergothérapeute constate également qu'Hélène souhaite (2) «aller dehors, voir la nature ", (3) assumer son rôle d'hôtesse envers ses visiteurs fréquents et (4) se maquiller «pour avoir l'air moins malade». De plus, (5) des plaies de pression (stade 1) sont présentent à la région du sacrum et sur les deux talons, ce qui lui occasionne de la douleur durant la nuit. Enfin, son conjoint a (6) peur qu'Hélène tombe lorsqu'il l'assiste pour l'habillage ou lorsqu'il l'aide à se transférer, par exemple entre le fauteuil roulant et le lit ou la toilette.

Le couple est toutefois catégorique sur deux points: premièrement, bien qu'un lit d'hôpital avec un matelas thérapeutique faciliterait les soins et préserverait l'intégrité de la peau d'Hélène, ils souhaitent dormir ensemble jusqu'au décès. Deuxièmement, les relations avec le propriétaire du logement sont tendues et aucun changement permanent ne doit être fait dans leur domicile.

Hélène, son conjoint et l'ergothérapeute conviennent donc d'un plan de traitement pour la prochaine semaine. Pour l'atteinte de six objectifs occupationnels énumérés ci-dessous, trois visites à domicile d'une durée d'environ une heure sont effectuées. Les modalités d'interventions sont l'attribution d'aides techniques en prêt de la flotte du service de soutien à domicile de leur centre de santé et des services sociaux, des conseils et de l'enseignement à Hélène et ses proches sur les techniques de soins, des mises en situation et de l'entraînement occupationnel.

Après le décès d'Hélène, six semaines plus tard, son conjoint remercie l'équipe du service de soutien à domicile. Dans la carte, il écrit: «Merci d'avoir permis à Hélène de faire des petites choses qui voulaient dire beaucoup pour elle.» 


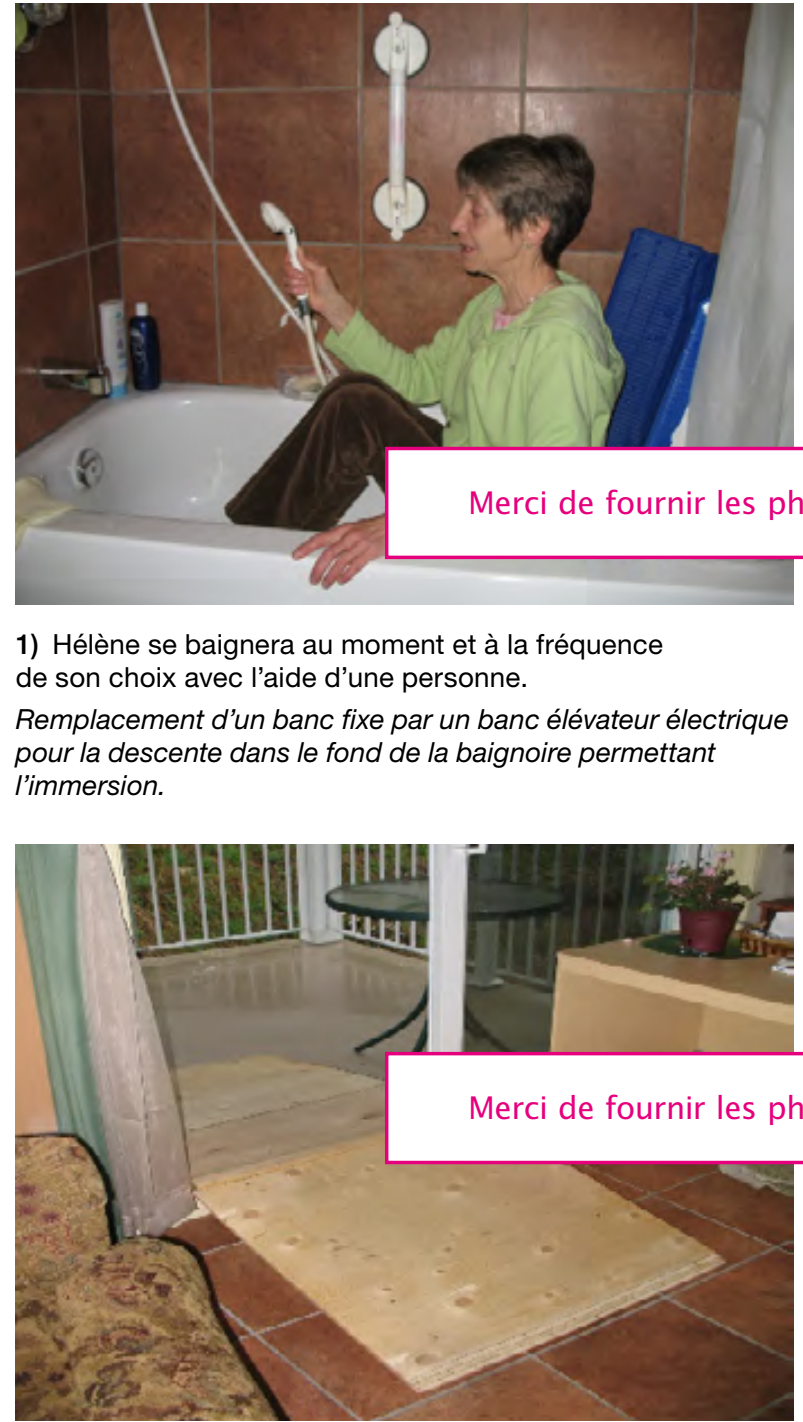

2) Hélène accédera seule au balcon en fauteuil roulant. Consignes transmises au frère pour la construction d'un biseau amovible pour franchir le seuil de la porte.

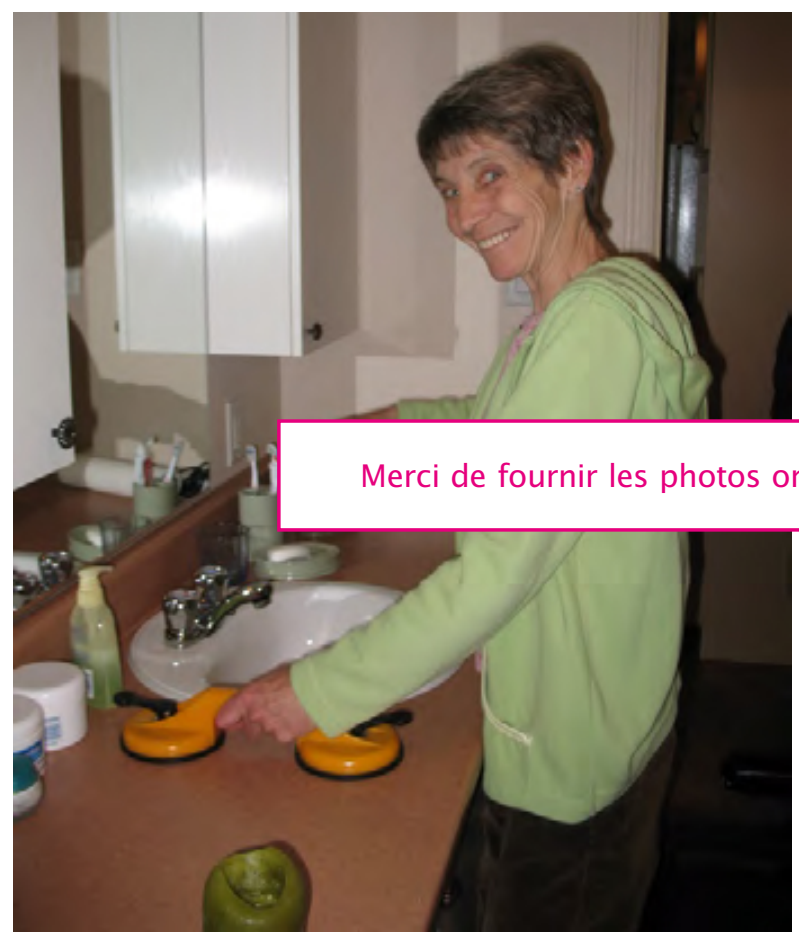

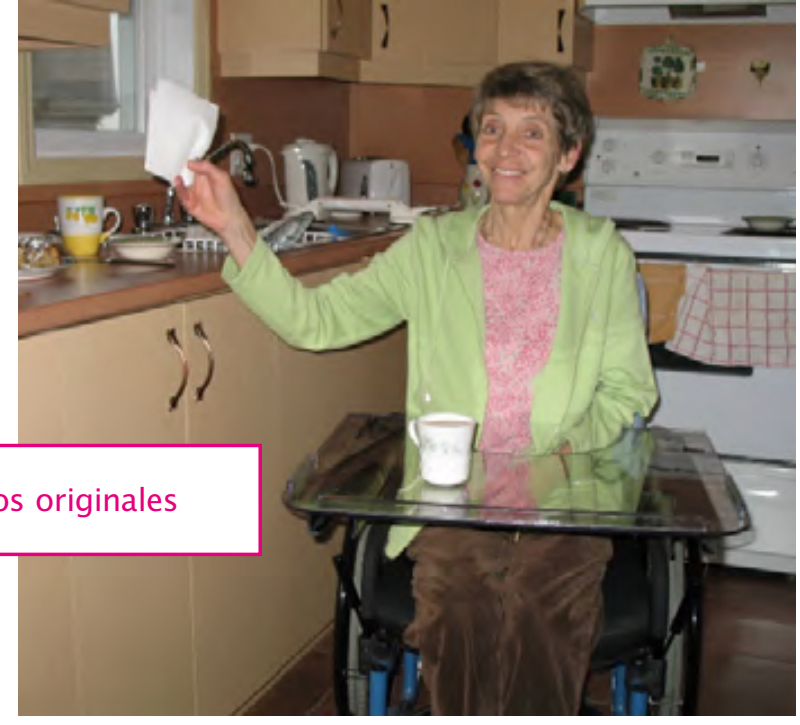

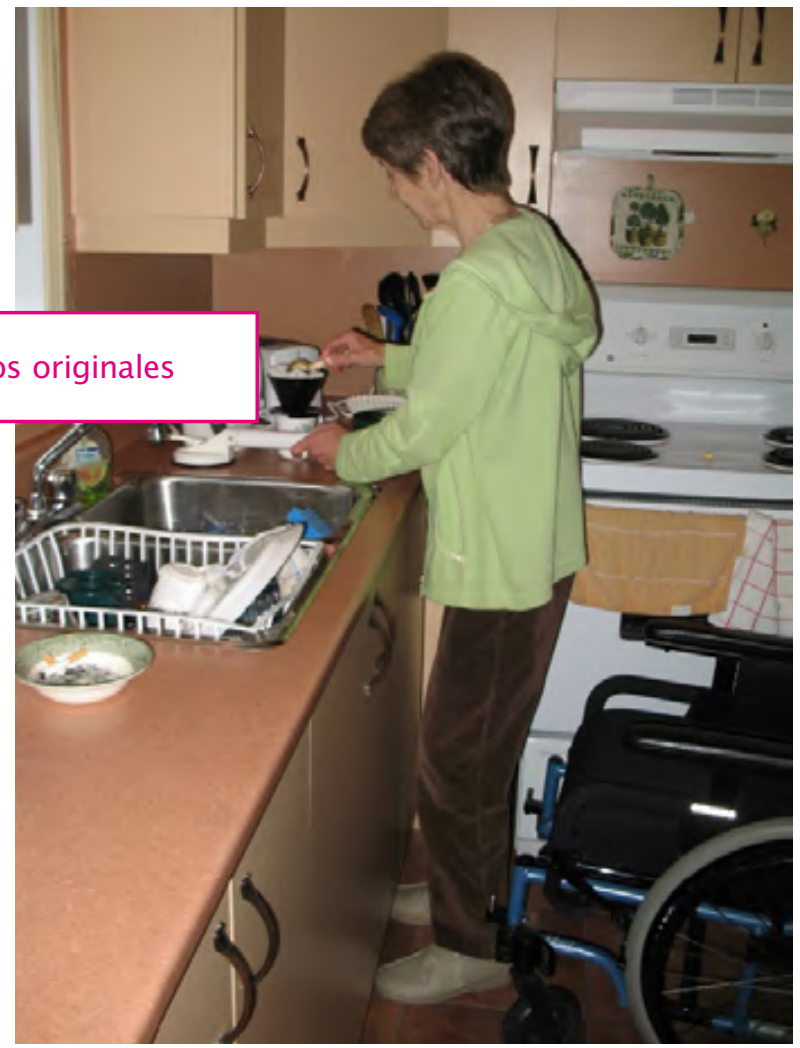

3) Hélène préparera seule des breuvages chauds et des collations afin de les servir seule à ses invités.

Ajout d'une poignée ventouse pour un appui sécuritaire en station debout lors de la préparation des aliments.

Ajout d'un plateau pour fauteuil roulant pour le transport
4) Hélène se maquillera lorsqu'elle le désirera Ajout d'une poignée ventouse pour un appui lors du maquillage nécessitant de fléchir le tronc en station debout pour s'approcher du miroir. 


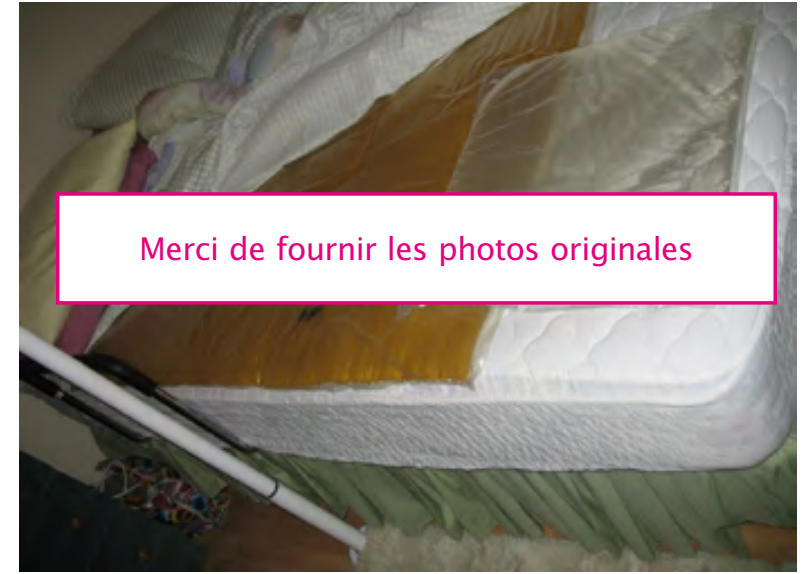

5) Hélène dormira avec son conjoint sur une surface thérapeutique pour prévenir l'aggravation des plaies et soulager ses douleurs.

Ajout d'un matelas de gel et d'un coussin d'eau aux talons, sous le drap, dans le lit double.

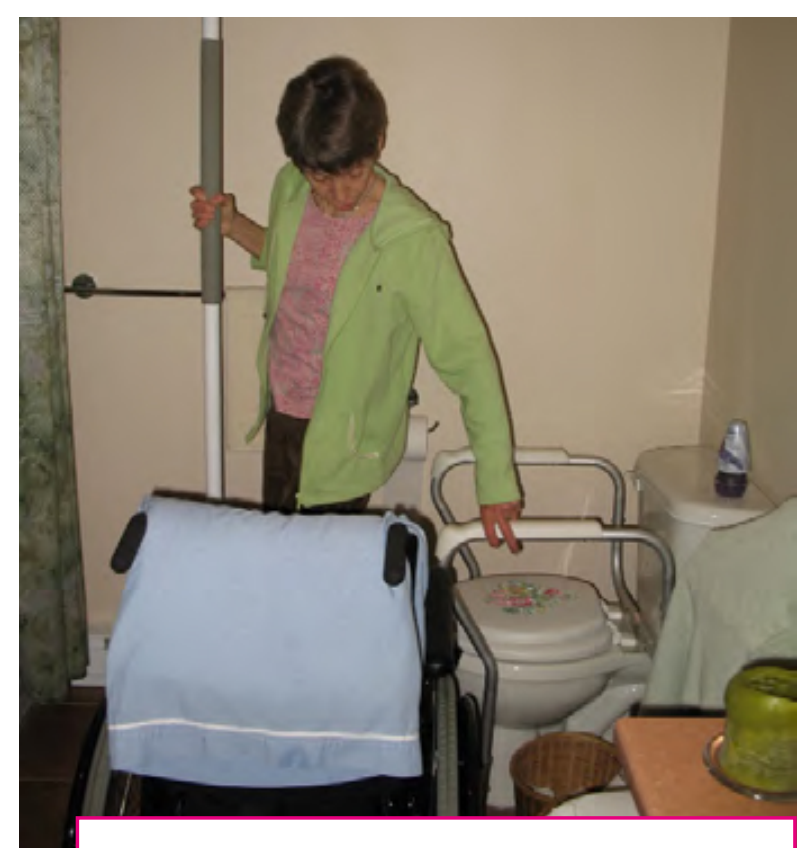

Merci de fournir les photos originales

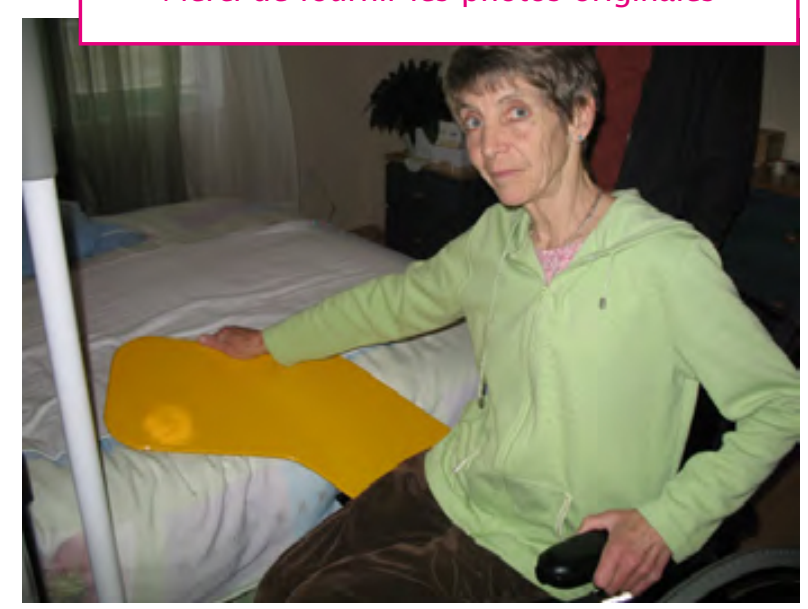

6) Hélène se transférera avec l'aide minimale d'une personne à la toilette et au lit.

Ajout d'appuis, soit un poteau plancher-plafond et un cadre sécuritaire à la toilette.

Ajout d'une planche de transfert et d'un poteau plancherplafond dans la chambre.

\section{Discussion}

Lergothérapie a pour objectif de promouvoir la participation de la personne dans des occupations signifiantes, dans l'environnement qui lui est propre [14]. Pour l'ergothérapeute, le bien-être des personnes passe par la participation à des occupations donnant un sens à leur vie [15,16]. Wilcock et Hocking proposent que l'occupation englobe non seulement ce qui est significatif, mais toutes les choses qu'une personne veut, doit, ou a besoin de faire, que ce soit pour elle-même ou pour la collectivité, afin de veiller à son bien-être et sa sécurité, ou à celle d'autrui [16].

Lorsque la mort est annoncée, les occupations donnant un sens à l'existence sont bouleversées. Les personnes atteintes d'une maladie potentiellement terminale vivent une privation progressive d'occupations [17], et il pourrait en résulter une souffrance existentielle. Bien qu'une définition consensuelle du concept de souffrance existentielle ne semble pas disponible [18], Bates a identifié que cet état est lié à, notamment, un manque de sens dans la vie au jour le jour, et une perte d'engagement dans les activités, les relations et les rôles [19]. Or, le but de l'ergothérapie est de soutenir les personnes dans la participation à des occupations porteuses de sens, avec leurs proches et dans leurs lieux de vie.

Le maintien (ou le retour) à domicile peut être un moyen pour permettre à la personne de réaliser ces occupations signifiantes avant sa mort. L'ergothérapeute peut soutenir les individus (ex. personnes en fin de vie, proches, membres de l'équipe interdisciplinaire) au regard du lieu choisi pour vivre une fin de vie digne, incluant le domicile [12]. L'ergothérapeute est en mesure d'évaluer les possibilités pour une personne de demeurer (ou retourner) en sécurité chez elle, malgré des pertes d'autonomie importantes [11]. De plus, les connaissances médicales de l'ergothérapeute lui permettent d'anticiper le déclin fonctionnel associé à l'évolution de la maladie et de proposer une organisation du quotidien adaptée à cette fin de vie [11]. L'ergothérapeute maîtrise l'influence de l'environnement sur le fonctionnement humain.

Les occupations des personnes en fin de vie changent en fonction du chez-soi de chacun, et de la composition plurielle de leur réseau d'aidants, avec des proches ou des travailleurs. D'une part, l'aménagement domiciliaire influence la réalisation des occupations. Par exemple, pour se laver, il est possible de se doucher debout dans une cabine ou bien de s'immerger dans l'eau d'une baignoire. Des capacités 
physiques distinctes sont nécessaires pour ces deux occupations visant l'hygiène corporelle, dans deux environnements différents. La préférence d'une personne en fin de vie devrait être considérée dans les façons de faire ses occupations, et des moyens devraient être mis en œuvre pour respecter ce choix. D'autre part, si certaines personnes sont entourées de proches disposés à assumer des soins et des services, d'autres ont recours à du personnel rémunéré. Les connaissances sur les techniques de prestation des soins et des équipements disponibles pour faciliter la réalisation des tâches varient significativement entre ces deux types d'aidants. Les uns comme les autres ont besoin d'assurer leur sécurité et le respect de leurs valeurs dans l'accomplissement de leur rôle. L'ergothérapeute est tout désigné pour favoriser l'atteinte de ces buts.

Pour ce faire, trois sphères centrales à la pratique de l'ergothérapie sont ciblées: la personne $(\mathrm{P})$, l'environnement $(\mathrm{E})$ et l'occupation $(\mathrm{O})$. L'ergothérapeute contribue ainsi à minimiser les situations de handicap, soit en augmentant les capacités physiques, cognitives, ou émotionnelles de la personne $(\mathrm{P})$, soit en modifiant l'environnement pour minimiser les barrières à la participation (E), ou encore en modulant l'occupation, tout en maintenant son sens $(\mathrm{O})$. Léducation, les modifications à l'environnement et l'attribution d'équipements spécialisés ou d'aides techniques sont des modalités pouvant être mises en œuvre pour atteindre ses objectifs [11-13].

Par exemple, un ergothérapeute cible le meilleur endroit pour installer le lit de la personne mourante, en considérant ses désirs et ceux de ses proches, et suggère la surface thérapeutique qui préviendra les plaies de pression, tout en facilitant la participation à des activités de socialisation et de réminiscence. Ou encore, l'ergothérapeute enseigne les méthodes compensatoires aux proches et propose des aides techniques pour réaliser l'habillage, l'alimentation ou les soins personnels en fonction des préférences de la personne. Aussi, l'ergothérapeute propose un aménagement du domicile, incluant les équipements, le cas échéant, pour des manœuvres et des déplacements sécuritaires de la personne en fin de vie, minimisant les risques de blessures musculosquelettiques chez l'aidé et l'aidant, dans l'environnement exigu que constituent souvent les pièces du domicile.

En somme, l'ergothérapeute habilite la personne à faire ce qu'elle souhaite, en fonction de ses (in)capacités, ainsi que de son environnement humain et physique. Alors que certains ergothérapeutes ciblent l'autonomie fonctionnelle et la réalisation d'activités afin de donner de la valeur à la vie durant le temps qu'il reste [21], d'autres rappellent l'importance des notions d'activités signifiantes (sens individuel de l'activité) et significatives (sens social) en cancérologie [20]. Ce sont les premières, les activités signifiantes, qui devraient être au cœur des pratiques professionnelles auprès des personnes. En outre, Guay et coll. avancent que «la valeur de la vie» est une vision restrictive des parcours en soins palliatifs: la mort intentionnelle est aussi une occupation humaine [22]. En effet, l'engagement dans une demande d'aide médicale à mourir peut être une occupation signifiante, soit une activité qu'une personne planifie et qu'elle souhaite mettre en œuvre pour le sens qu'elle revêt [23]. Ainsi, l'ergothérapeute soutient l'organisation de la vie quotidienne de la personne pour que sa vie et sa mort à la maison soient signifiantes et qu'elles se réalisent en cohérence avec ses aspirations.

\section{Conclusion}

Laccompagnement et le soutien de la personne qui souhaite mourir à domicile devraient inclure l'ergothérapeute, puisque son expertise favorise le maintien (ou le retour) à la maison jusqu'au décès, dans le respect des volontés de la personne qui le désire. L'ergothérapeute optimise l'organisation du quotidien, tout en facilitant une participation de la personne à des occupations donnant du sens à ses derniers jours, en fonction des (in)capacités, dans un environnement humain et physique qui lui est propre. Aider la personne en fin de vie pour qu'elle maintienne une participation à ses occupations signifiantes devrait être central dans les soins et les services offerts, notamment pour contrer la souffrance existentielle. 


\section{Références bibliographiques}

1. Gomes B, Higginson IJ, Calanzani N, Cohen J, Deliens L, Daveson BA et Menaca A (2012). Preferences for Place of Death if Faced with Advanced Cancer: A Population Survey in England, Flanders, Germany, Italy, the Netherlands, Portugal and Spain. Annals of Oncology. 23(8): 2006-2015.

2. Gomes B, Calanzani N, Gysels M, Hall S et Higginson IJ (2013). Heterogeneity and Changes in Preferences for Dying at Home: A Systematic Review. BMC palliative care. 12(1): 7.

3. Pivodic L, Pardon K, Morin L, Addington-Hall J, Miccinesi G, Cardenas-Turanzas M et Wilson DM (2016). Place of Death in the Population Dying from Diseases Indicative of Palliative Care Need: A Cross-National Population-Level Study in 14 Countries. J Epidemiol Community Health. 70(1): 17-24.

4. Gagnon B, Nadeau L, Scott S, Dumont S, MacDonald N, Aubin M et Mayo N (2015). The Association between Home Palliative Care Services and Quality of End-of-Life Care Indicators in the Province of Quebec. Journal of pain and symptom management. 50(1): 48-58.

5. Raziee H, Saskin R et Barbera L (2017). Determinants of Home Death in Patients with Cancer: A Population-Based Study in Ontario, Canada. Journal of Palliative Care. 32(1): 11-18.

6. Hardy J, Maresco-Pennisi D, Gilshenan K et Yates P. (2008). Barriers to the Best Care of the Dying in Queensland, Australia. J Palliat Med. 11(10): 1325-1329. PM: 19115892

7. O'Brien M et Jack B. Barriers to Dying at Home: The Impact of Poor Co-Ordination of Community Service Provision for Patients with Cancer. Health Soc Care Community. 2010; 18(4): 337-345. PM: 20039968.

8. Gouvernement du Québec (2015). Soins palliatifs et de fin de vie. Plan de développement 2015-2020. Québec (Canada): La direction des communications du ministère de la Santé et des Services sociaux. Consulté le 25 novembre 2019 à l'adresse http://publications. msss.gouv.qc.ca/msss/fichiers/2015/15-828-01W.pdf

9. Leclerc B-S, Blanchard L, Cantinotti M, Couturier Y, Gervais D, Lessard S et Mongeau S (2014). The Effectiveness of Interdisciplinary Teams in End-of-Life Palliative Care: A systematic Review of Comparative Studies. Journal of Palliative Care, Montreal. 30(1): 44-54.

10. Association canadienne des ergothérapeutes (ACE) (2019). L'ergothérapie et les soins de fin de vie. Consulté le 19 octobre 2019 à l'adresse https://caot.in1touch.org/document/4101/End \%20of\%20 Life \%20Care \%20-\%20FS.pdf

11. Occupational Therapy Australia Ltd (OTAL) (2015). Position Statement: Occupational Therapy in Palliative Care. Australian Occupational Therapy Journal. 62(6): 459-461. https://doi.org/10.1111/1440-1630.12264
12. Society of Alberta Occupational Therapists (SAOT) (2016). The Role of Occupational Therapy (OT) in Medical Assistance in Dying. Consulté le 19 octobre 2019 à l'adresse www.saot.ca/wp-content/ uploads/2018/09/Role-of-Occupational-Therapy-in-Medical-Assistance-in-Dying.pdf

13. Gouvernement du Québec (2008). Plan directeur de développement des compétences des intervenants en soins palliatifs. Québec (Canada): La Direction des communications du ministère de la Santé et des Services sociaux. Consulté le 19 octobre 2019 à l'adresse https://publications.msss.gouv.qc.ca/msss/fichiers/2008/08-902-03.pdf

14. WFOT (2012). About occupational Therapy. Consulté le 3 septembre 2017 à l'adresse www.wfot.org/aboutus/aboutoccupationaltherapy/ definitionofoccupationaltherapy.aspx

15. Townsend EA et Polatajko HJ (2013). Habiliter à l'occupation. Faire avancer la perspective ergothérapique de la santé, du bien-être et de la justice par l'occupation ( $2^{\mathrm{e}}$ éd.). Ottawa, Publications ACE.

16. Wilcock AA et Hocking $C$ (2015). An occupational perspective of health ( $3^{\circ}$ éd.). Thorofare, NJ: SLACK Incorporated.

17. Lyons M, Orozovic N, Davis J et Newman J. Doing-Being-Becoming: Occupational Experiences of Persons with Life-Threatening IIInesses. American Journal of Occupational Therapy. 2000; 56(3): 285-295.

18. Boston P, Bruce A et Schreiber R. Existential Suffering in the Palliative Care Setting: An Integrated Literature Review. Journal of pain and symptom management. $2011 ; 41(3)$ : 604-618.

19. Bates AT. Addressing Existential Suffering. British Columbia Medical Journal. 2016; 58(5): 268-273

20. Soum-Pouyalet F, Sorita E et Belio C (2018). L'ergothérapie en cancérologie. In: Bataille P, Amsellem N. Cancers, entre biomédicalisation et (dé-)subjectivation. La Découverte, 341 p.

21. Gueguen AF et Bereziat MP. Ergothérapie en soins palliatifs. Ou comment promouvoir l'autonomie de la personne en fin de vie? INFOKara. 2002; 17(1): 20-23.

22. Guay M, Drolet MJ, Kuhne N, Talbot-Coulombe C et Mortenson B (3rd revision submitted in April 2020). Critically Reflecting on Deliberate Death as a Final Occupation. Journal of Occupational Science.

23. Guay M, Talbot-Coulombe $\mathrm{C}$ et Drolet MJ (Sous presse). Être l'ergothérapeute d'une personne demandant l'aide médicale à mourir. Occupation: Ergothérapeute. 